\title{
Evaluation of the Selfitis Behavior Scale Across Two Persian- Speaking Countries, Iran and Afghanistan: Advanced Psychometric Testing in a Large-Scale Sample
}

\author{
Lin, C.-Y. ${ }^{a}$, Lin, C.-K. ${ }^{\text {b }, ~ I m a n i, ~ V . c, ~ G r i f f i t h s, ~ M . D . ~}{ }^{\mathrm{d}}$, Pakpour, A.H.e,f
}

aDepartment of Rehabilitation Sciences, The Hong Kong Polytechnic University, Hung Hom, Hong Kong

${ }^{b}$ Department of Environmental Health, Harvard T.H. Chan School of Public Health, Boston, MA 02115, United States

'Pediatric Health Research Center, Tabriz University of Medical Sciences, Tabriz, Iran

'International Gaming Research Unit, Psychology Department, Nottingham Trent University, Nottingham, United Kingdom

eSocial Determinants of Health Research Center, Qazvin University of Medical Sciences, Shahid Bahonar Blvd, Qazvin, 3419759811, Iran

fDepartment of Nursing, School of Health and Welfare, Jönköping University, Jönköping, Sweden

\section{Abstract}

Selfitis - which started off as a hoax but has now been investigated empirically - has been defined as the obsessive-compulsive desire to take photos of oneself and post them on social media. Furthermore, a scale to assess selfitis, the Selfitis Behavior Scale (SBS), has been developed. This study applied advanced psychometric testing methods, including confirmatory factor analysis (utilizing classical test theory) and the Rasch model (utilizing modern test theory), to examine the psychometric properties among Persian speakers (in Iran and Afghanistan). The participants (3163 Iranians and 1100 Afghanistani) completed an online survey posted on Instagram pages. The SBS showed promising properties, including satisfactory reliability (e.g., internal consistency and test-retest reliability), excellent construct validity (e.g., good fit in the CFA and Rasch models), and acceptable measurement invariance across Iranian and Afghan samples. Consequently, the SBS is a valid and reliable instrument for assessing selfitis among Persian-speaking samples. 